\title{
Utility of biochemical tests in prediction, diagnostics and clinical management of preeclampsia: a review
}

\author{
Jakub Kornacki, Ewa Wender-Ożegowska
}

Division of Reproduction, Poznan University of Medical Sciences, Poznan, Poland

Submitted: 2 April 2018

Accepted: 25 July 2018

Arch Med Sci 2020; 16 (6): 1370-1375

DOI: https://doi.org/10.5114/aoms.2020.97762

Copyright @ 2020 Termedia \& Banach

\begin{abstract}
The most widely accepted theory for the development of preeclampsia is the "two-stage theory". An imbalance between antiangiogenic and proangiogenic factors is considered the link between the two stages. Nowadays, an increasing amount of data is available on the use of measurements of serum concentrations of these factors in the prediction, diagnosis and management of preeclampsia. The most useful, modern biochemical test that may help in making crucial clinical decisions in patients with preeclampsia is the sFlt-1/PIGF (soluble fms-like tyrosine kinase 1/placental growth factor) ratio. The aim of this review is to present the current use of different biochemical tests in the prediction, diagnosis and management of preeclampsia. Development of these diagnostic methods in recent years and a belief in their ground-breaking role in modern management of preeclampsia make this review especially important.
\end{abstract}

Key words: preeclampsia, endothelium, placenta, proangiogenic markers, antiangiogenic markers, soluble fms-like tyrosine kinase 1, placental growth factor.

\section{Introduction}

In spite of considerable research, preeclampsia still remains probably the most mysterious and interesting complication of pregnancy. It affects $2-3 \%$ of pregnant women, mostly nulliparous patients [1]. Although the common definition of preeclampsia, including the new onset of hypertension and proteinuria in the second half of pregnancy, is still widely accepted, different societies have modified certain criteria of the disease [1-3]. In 2013, the American College of Obstetricians and Gynecologists (ACOG) decided not to necessarily include proteinuria in the diagnosis of preeclampsia [2]. According to them, preeclampsia may be diagnosed if new onset of hypertension after 20 weeks of pregnancy is associated with at least one of the following: 1) thrombocytopenia 2) renal failure, 3) liver failure, 4) pulmonary edema or 5) neurological and/or visual disturbances [2]. Similarly, the Society of Obstetricians and Gynecologists of Canada (SOGC) decided that proteinuria is not an essential criterion of preeclampsia, which may be diagnosed also if the presence of hypertension in the second half of pregnancy is accompanied by a different complication or adverse condition [3]. They included, besides the complications mentioned above by ACOG, the consequences of feto-placental disturbances, such as intrauterine growth restriction (IUGR), oligohy-

\author{
Corresponding author: \\ Jakub Kornacki PhD \\ Division of Reproduction \\ Poznan University \\ of Medical Sciences \\ 33 Polna St \\ 60-535 Poznan, Poland \\ Phone: +48618419302 \\ E-mail: kuba.kornacki@wp.pl
}


dramnios, and abnormal results of the fetal umbilical artery and/or ductus venosus Doppler [3].

These modifications indicate the complex and multiorgan nature of the disease as well as the crucial role of the placenta in the pathophysiology of preeclampsia. Such an approach should increase the diagnostic accuracy.

Currently, the most widely accepted theory for the development of preeclampsia is the "twostage theory" [4]. According to this theory, the first stage of the disease is reduced placental perfusion and the second is generalized maternal endothelium dysfunction [4]. The main cause of reduced placental perfusion is poor placentation as a consequence of impaired trophoblast invasion into the lumen and the walls of spiral arteries. Endothelium damage is probably caused by antiangiogenic factors excessively produced by the poorly perfused placenta and transferred to the maternal circulation [4]. These factors, including soluble fms-like tyrosine kinase 1 (sFlt1) and soluble endoglin (sEng), contribute to endothelium damage and dysfunction by binding, respectively, vascular endothelial growth factor (VEGF) and transforming growth factor (TGF)- $\beta 1$, which are required to maintain normal endothelium function [5]. The consequence of maternal endothelium damage may be the different symptoms and complications characteristic of preeclampsia, such as hypertension, neurological symptoms, proteinuria and thrombocytopenia. The increased production of antiangiogenic factors in the placentas of patients who developed complications was observed many weeks before the onset of the disease [6].

This "two-stage theory" for the development of preeclampsia mainly explains the pathophysiology of the early-onset disease that develops before 34 weeks of gestation, whereas late-onset preeclampsia (> 34 weeks), including term preeclampsia ( $\geq 37$ weeks), may have a different etiology [7]. The late-onset disease may be attributed to the primary cardiovascular system pathology, including angiopathy and/or decreased cardiac output without the primary role of poor placentation in the onset of the disease $[7,8]$. The possible risk factors of this form of preeclampsia include pregestational diabetes, particularly class $\mathrm{F}$ or $\mathrm{R}$, hyperlipidemia, obesity and chronic hypertension [7, 9].

The purpose of this review was to summarize and emphasize the role of the serum assessment of many different popular biochemical markers, including new ones, in the prediction, diagnostics and clinical management of preeclampsia. The recent enormous scientific development in this field seems to be critical for the change in clinical management of preeclampsia in the future. This is why it seems important to bring this topic closer to the attention of clinicians.

\section{Material and methods}

\section{Search process}

The Medline (PubMed) database was searched from January 2004 to April 2018 to identify articles on use of biochemical tests in preeclampsia. There was no restriction on language. The following key words and subject headings were used in the search: "preeclampsia", "biochemical tests", "antiangiogenic factors" , sFlt-1, soluble endoglin (sEng), PIGF, pregnancy-associated plasma protein A (PAPP-A) and placental protein 13 (PP 13).

\section{Inclusion criteria}

The following inclusion criteria were applied:

- full text articles assessing the value of biochemical tests in prediction of preeclampsia in first, second and third trimesters of pregnancy,

- full text articles assessing the value of biochemical tests in diagnosis of preeclampsia,

- full text articles assessing the value of biochemical tests in management of preeclampsia,

- full text articles assessing the value of biochemical tests in monitoring of preeclampsia treatment by new methods.

Studies were included if they reported original data only.

\section{Exclusion criteria}

Articles were excluded from the final review if: - they concerned not only preeclampsia but other types of hypertension in pregnancy or other pregnancy pathologies,

- they, as full text articles, were written in a language other than English or Polish,

- they did not analyze the prediction value of biochemical tests in a specific period of time at the first trimester (11-13 weeks gestation).

\section{Results}

Of the 92 studies identified, 24 were included in our review. Details on the study selection process are shown in the Figure 1.

\section{Quality of included studies}

All included studies were prospective. All studies had adequately described population selection, tests, measurement used, and outcomes. In the studies concerning prediction of preeclampsia in first trimester all recruited women were between 11 and 13 weeks gestation.

\section{Use of biochemical markers in preeclampsia prediction in the first trimester of pregnancy}

Nowadays, as in the case of fetuses with trisomies, we can almost accurately predict the oc- 


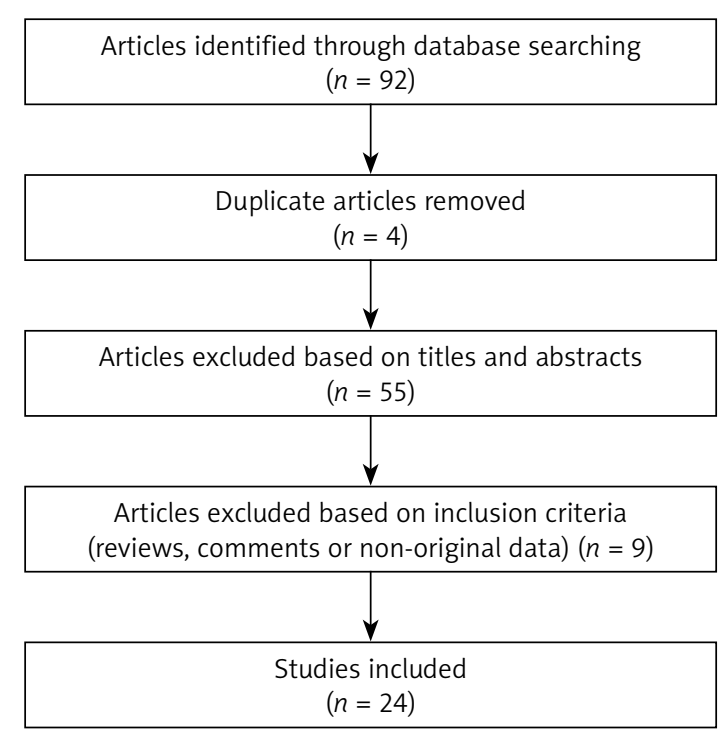

Figure 1 . Study selection process

currence of preeclampsia in early pregnancy [6, 10]. The most popular first trimester biochemical markers of preeclampsia are as follows: 1) pregnancy-associated plasma protein A, 2) placental growth factor (PIGF), 3) soluble fms-like tyrosine kinase 1 (sFlt1), 4) placental protein 13 (PP13), and 5) triglycerides $[10,11]$.

Pregnancy-associated plasma protein A (PAPP-A) is an enzyme, a metalloproteinase, produced by syncytiotrophoblasts, responsible for the cleavage of complex insulin-like growth factor (IGF)-binding protein (IGFBP). The consequence of its activity is release of IGF from the complex $[12,13]$. IGF stimulates placental growth and development [14]. In the first trimester of pregnancies complicated later by preeclampsia, the serum concentration of PAPP-A has been found to be significantly lower than that in pregnancies in which preeclampsia did not develop [10, 11]. In clinical practice the serum level of PAPP-A should be presented in percentiles or in multiples of median (MoM) [11, 14]. Further, the decreased concentration of PAPP-A at $11-13$ weeks of pregnancy has a $44 \%$ and $37 \%$ detection rate of early and late preeclampsia, respectively, with a false positive rate (FPR) of $5 \%$ $[15,16]$.

Placental growth factor (PIGF) is a protein, a vascular endothelial growth factor (VEGF) homolog, produced by cytotrophoblasts. Similar to VEGF, it has proangiogenic activity, and thus promotes placental growth and development $[14,17]$. The maximum serum concentration of PIGF is observed at 29-32 weeks of pregnancy [17]. In pregnancies complicated by preeclampsia the PIGF level in the first trimester of pregnancy decreased [18-20]. In clinical practice, the serum concentration of PIGF is expressed in percentiles or in MoM. The use of PIGF alone in the prediction of preeclampsia in the first trimester of pregnancy has a detection rate of $59 \%$ and $41 \%$ for early and late preeclampsia, respectively, with a false positive rate of $5 \%[15,16]$.

Soluble fms-like tyrosine kinase 1 (sFlt1) is a circulating protein and a soluble form of the vascular endothelial growth factor 1 receptor (VEGFR1), produced by syncytiotrophoblasts [5]. It is an antiangiogenic factor that inhibits proangiogenic activity of VEGF, including its protective effect on the endothelium. It may bind both PIGF and VEGF, which has a negative influence on the placenta and the maternal endothelium [5]. In patients who later developed preeclampsia the serum concentration of sFlt-1 in the first trimester was significantly higher than in pregnant women, who remained healthy throughout their pregnancy [21].

Placental protein 13 (PP13) is a protein produced by trophoblasts. It is an immunomodulator that induces the apoptosis of T cells and macrophages, and plays an important role in the early placental and maternal-placental interface development [22-24]. As for PAPP-A and PIGF, in patients who developed preeclampsia, the serum level of PP13 was significantly lower than that in patients who remained normotensive throughout their pregnancy $[11,25]$. In a meta-analysis, a low level of PP13 in the first trimester of pregnancy was found to be more predictive for the occurrence of early preeclampsia than that of late preeclampsia [11].

The common feature of all of the above biochemical markers of preeclampsia (PAPP-A, PIGF, sFlt-1, and PP 13) is their placental origin. Other, non-placental, markers of preeclampsia development are triglycerides.

The use of biochemical tests in the first trimester of pregnancy together with the other methods, including ultrasound, has been found to be very effective in the prediction of preeclampsia, mainly early onset or preterm preeclampsia [10, 14]. Nowadays it is well accepted to use the measurement of PAPP-A and PIGF together with the assessment of the mean uterine artery pulsatility index (PI) and the mean arterial pressure (MAP) at 11-13 weeks of pregnancy in the screening of preeclampsia $[10,14]$. Using this method, we can predict early-onset preeclampsia ( $<34$ weeks) and preterm preeclampsia (< 37 weeks) in $93 \%$ and $61 \%$ of cases, respectively, with a false positive rate of $5 \%[15,16]$. At the same time, the prediction of term preeclampsia ( $>37$ weeks) is considerably poor (only $38 \%$ ) $[15,16]$.

\section{Prediction of preeclampsia in the second and third trimester of pregnancy using biochemical markers}

In the second half of pregnancy, we can predict the development of preeclampsia by assessing 
the serum level of angiogenic and antiangiogenic markers including PIGF, sFlt-1, and soluble endoglin (sEng). The increased levels of serum sFlt-1 and sEng were found to be significantly higher in the second and third trimester of pregnancy in patients who developed preeclampsia than in pregnant women who remained healthy throughout their pregnancy $[26,27]$. This correlation mainly concerned preterm preeclampsia [26, 27]. Conversely, those women who developed preeclampsia had significantly lowers levels of PIGF in the second and third trimesters than patients who remained normotensive throughout their pregnancy $[26,27]$. The levels of PIGF in women who developed preterm preeclampsia were lower than in patients with the term onset of the disease [27]. In one of the studies, the highest predictive value, particularly for the development of preterm preeclampsia, was found for sFlt-1/PIGF ratio [26]. The decreased or increased concentration of the angiogenic or antiangiogenic factors, respectively, is found usually already 5-6 weeks before the onset of the disease [26]. The same phenomenon is even more evident for the sFlt-1/PIGF ratio, which increases a few weeks before the presentation of the disease. Therefore, the sFlt-1/PIGF ratio may be regarded as an index of angiogenic imbalance [28]. PROGNOSIS study showed a very high negative predictive value (NPV) of $99.3 \%$ for the occurrence of preeclampsia within a week if the sFlt-1/ PIGF ratio was $\leq 38$ and a positive predictive value (PPV) of $36.7 \%$ for the onset of the disease within 4 weeks if the ratio was $>38$ [29]. A summary of the use of different biochemical markers in preeclampsia is presented in the Table I.

\section{Use of biochemical tests in preeclampsia diagnosis}

At present, in spite of the considerable development in the research and clinical practice regarding preeclampsia, the diagnosis of this disease is still sometimes difficult. It is usually associated with a heterogenic clinical presentation and the sudden onset of the disease. In many cases not all maternal organs are involved in the pathophysiology of preeclampsia. Some patients present with more placenta-related symptoms, and others with more maternal ones [7]. Thus, according to some gynecological associations, now it is not necessary to include proteinuria as a criterion of the disease [2, 3].

The significant progress in the efficacy of the diagnosis of preeclampsia, a particularly atypical form of the disease or the related complications, may be the use of the serum measurement of the angio- and antiangiogenic markers, including PIGF and sFlt-1. The most accurate one of these markers is the sFlt-1/PIGF ratio [6, 30, 31]. Before 34 weeks of gestation, an sFlt-1/PIGF ratio of $\geq 85$ was found to have a sensitivity/specificity of $88 \% / 99.5 \%$ in the diagnosis of preeclampsia [30]. The above ratio also predicts the occurrence of maternal and fetal complications of preeclampsia within 2 weeks very well [32]. At $\geq 34$ weeks of gestation, the highest sensitivity/specificity was found for the sFlt-1/ PIGF ratio of $\geq 110$ (58.2 and $95.5 \%$, respectively) [30]. The measurement of the sFlt-1/PIGF ratio also plays an important role in ruling out preeclampsia $[6,30]$. Both before and after 34 weeks, if the ratio is $\leq$ 33 , the sensitivity/specificity for ruling out of preeclampsia is very high, 95\%/94\% ( $<34$ weeks) and 89.6\%/73.1\% ( $\geq 34$ weeks) [30].

Additionally, the sFlt-1/PIGF ratio measurement may be helpful in the diagnosis of different subforms of preeclampsia, such as the HELLP syndrome, as well as the differential diagnosis of HELLP with other causes of thrombocytopenia during pregnancy [33].

\section{Use of biochemical tests in preeclampsia management}

Although preeclampsia is very often a dangerous condition for both a mother and a fetus, the severity of the disease varies. Besides the degree of hypertension and proteinuria if present, the degree of placental insufficiency, and the coexistence of different complications of preeclampsia, including hepatic or neurological ones, another possible marker of the intensity of the disease is the sFlt-1/PIGF ratio [34]. For example, in patients with preeclampsia in whom the abovementioned ratio was $<85$ only $15.8 \%$ required delivery within 2 weeks in comparison to $86 \%$ of women with a ratio $>85$ [32]. This finding indicated a positive correlation between the ratio and the severity of the disease. The results of another study, which showed that if the sFlt-1/PIGF ratio was very high, above 655 , only $5.9 \%$ of the patients remained undelivered within a week, which confirmed the abovementioned findings [35]. These as well as other results showed the significant utility of this

Table I. Different biochemical markers and their levels in preeclampsia during first and later trimesters

\begin{tabular}{|lcc|}
\hline Biochemical marker & $\mathbf{1}^{\text {st }}$ trimester & $\begin{array}{c}2^{\text {nd }} \text { and } 3^{\text {rd }} \\
\text { trimester }\end{array}$ \\
\hline PAPP-A & $\downarrow$ & \\
\hline PIGF & $\downarrow$ & $\downarrow$ \\
\hline SFlt-1 & $\uparrow$ & $\uparrow$ \\
\hline PP13 & $\downarrow$ & $\uparrow$ \\
\hline Triglycerides & $\uparrow$ & $\uparrow$ \\
\hline SEng & $\uparrow$ & $\uparrow$ \\
\hline SFlt-1/PIGF & & \\
\hline
\end{tabular}


biochemical test, including also the need for hospitalization $[32,36]$. In the first study on this topic, the sFlt-1/PIGF ratio was proven to be a helpful clinical marker for determining whether to hospitalize a patient with preeclampsia or not [36]. Another helpful role of an assessment of this ratio is in determining the occurrence of both maternal and fetal complications during the course of preeclampsia. A significantly high sFlt-1/PIGF ratio was found in women with preeclampsia who developed different complications of the disease [32].

Currently, the most important utility of the assessment of the sFlt-1/PIGF ratio in women with preeclampsia seems to be ruling out the disease [34]. Therefore, it has already been implemented in clinical practice in some European countries, such as Germany, England and Italy [34]. It seems to be particularly helpful in various types of hypertensive disorders of pregnancy, idiopathic cases of placental insufficiency, and different dysfunctions of the liver and different causes of thrombocytopenia. All these pathologies may be a clinical element of preeclampsia.

\section{Use of sFlt-1/PIGF ratio in the monitoring of preeclampsia treatment by new methods}

One possible new treatment of preeclampsia is apheresis [37, 38]. The aim of this treatment is to remove sFlt-1 from the maternal circulation. The primary efficacy of the treatment with a significant, transient reduction of the sFlt-1/PIGF ratio was found [38].

\section{Conclusions}

Nowadays, various biochemical tests play a crucial role in the prediction of preeclampsia. Both in the first trimester and in the later trimesters, they are very helpful in predicting preeclampsia, particularly in the cases of an early onset of the disease. In the first trimester, both PAPP-A and PIGF assessments, together with other prediction methods, form the basis of a prediction test for preeclampsia. In the second and third trimesters the most helpful in the prediction of the disease seems to be the sFlt-1/PIGF ratio. Recently, the measurement of this ratio has gained increasing popularity not only as a prediction test but also as a diagnostic test with some utility in the management of preeclampsia.

\section{Conflict of interest}

The authors declare no conflict of interest.

\section{References}

1. Roberts J, Person G, Cutler J, Lindheimer M. Summary of the NHLBI Working Group on research on hypertension during pregnancy. Hypertension 2003; 41: 437-45.
2. American College of Obstetricians and Gynecologists. Hypertension in pregnancy. Report of the American College of Obstetricians and Gynecologists' Task Force on Hypertension in Pregnancy. Obstet Gynecol 2013; 122: 1122-31.

3. Magee LA, Pels A, Helewa M, Rey E, von Dadelszen P. Diagnosis, evaluation, and management ofthe hypertensive disorders of pregnancy: executive summary. J Obstet Gynecol Can 2014; 36: 416-38.

4. Roberts JM, Hubel CA. The two stage model of preeclampsia: variations on the theme. Placenta 2009; 30: S32-7.

5. Karumanchi SA. Angiogenic factors in preeclampsia. From diagnosis to therapy. Hypertension 2016; 67: 1072-9.

6. Schrey-Petersen S, Stepan H. Anti-angiogenesis and preeclampsia in 2016. Curr Hypertens Rep 2017; 19: 6.

7. Kornacki J, Skrzypczak J. Preeclampsia - two manifestations of the same disease. Ginekol Pol 2008; 79: 432-7.

8. Tobinaga CR, Torloni MR, Gueuvoghlanian-Silva BY, et al. Angiogenic factors and uterine Doppler velocimetry in early- and late-onset preeclampsia. Acta Obstet Gynecol Scand 2014; 93: 469-76.

9. Gutaj P, Zawiejska A, Mantaj U, Wender-Ożegowska E. Determinants of preeclampsia in women with type 1 diabetes. Acta Diabetol 2017; 54: 1115-21.

10. Poon CY, Kametas NA, Maiz N, Akolekar R, Nicolaides $\mathrm{KH}$. First-Trimester prediction of hypertensive disorders in pregnancy. Hypertension 2009; 53: 812-8.

11. Zhong Y, Zhu F, Ding Y. Serum screening in first trimester to predict pre-eclampsia, small for gestational age and preterm delivery: systematic review and meta-analysis. Pregnancy Childbirth 2015; 15: 191.

12. Bonno M, Oxvig C, Kephart GM, et al. Localization of pregnancy-associated plasam protein-A and colonization of pregnancy-associated plasma protein-A messenger ribonucleic acid and eosinophil granule major basic protein messenger ribonucleic acid in placenta. Lan Invest 1994; 71: 560-6.

13. Lawrence JB, Oxvig C, Overgaard MT, et al. The insulin-like growth factor (IGF)-dependent IGF binding protein-4 protease secreted by human fibroblasts is pregnancy-associated plasma protein-A. Proc Natl Acad Sci USA 1999; 96: 3149-53.

14. Poon LC, Nicolaides KH. First- trimester maternal factors and biochemical screening for preeclampsia. Prenat Diagn 2014; 34: 618-27.

15. Wright D, Akolekar R, Syngelaki A, Poon LC, Nicolaides $\mathrm{KH}$. A competing risks model in early screening for preeclampsia. Fetal Diagn Ther 2012; 32: 171-7.

16. Akolekar R, Syngelaki A, Poon L, Wright D, Nicolaides $\mathrm{KH}$. Competing risks model in early screening for preeclampsia by biophysical and biochemical markers. Fetal Diagn Ther 2013; 33: 8-15.

17. Powe CE, Levine RJ, Karumanchi SA. Preeclampsia, a disease of the maternal endothelium: the role of antiangiogenic factors and implications for later cardiovasular disease. Circulation 2011; 123: 2856-69.

18. Thadani R, Mutter WP, Wolf $M$, et al. First trimester placental growth factor and soluble fms-like tyrosine kinase 1 and risk for preeclampsia. J Clin Endocrinol Metab 2004; 89: 770-5.

19. Akolekar R, Zaragoza E, Poon LC, Pepes S, Nicolaides $\mathrm{KH}$. Maternal serum placental growth factor at $11+0$ to $13+6$ weeks of gestation in the prediction of pre-eclampsia. Ultrasound Obstet Gynecol 2008; 32: 732-9.

20. Crispi F, Llurba E, Dominguez C, Martin-Gallan P, Grata$\cos E$. Predictive value of angiogenic factors and uterine 
artery Doppler for early - versus late-onset preeclampsia and intrauterine growth restriction. Ultrasound $\mathrm{Ob}$ stet Gynecol 2008; 31: 303-9.

21. Crovetta F, Figueras F, Triunfo S, et al. First trimester screening for early and late preeclampsia based on maternal. Characteristics, biophysical parameters, and angiogenic factors. Prenat Diagn 2015; 35: 183-91.

22. Cerdeira AS, Kopcow HD, Karumanchi SA. Regulatory T cells in preeclampsia: some answers, more questions? Am J Pathol 2012; 181: 1900-2.

23. Than NG, Romero R, Kim CJ, Mc Gown MR, Papp Z. Wildman DE. Galactins: guardians of eutherian pregnancy at the maternal-fetal interface. Trends Endocrinol Metab 2012; 23: 23-31.

24. Than NG, Romero R, Goodman M, et al. A primate subfamily of galectins expressed at the maternal-fetal interface that promote immune cell death. Proc Natl Acad Sci USA 2009; 106: 9731-6.

25. Meiri H, Sammar M, Herzog A, et al. Prediction of preeclampsia by placental protein 13 and background risk factors and its prevention by aspirin. J Perinat Med 2014; 42: 591-601.

26. Noori M, Donald AE, Angelakopoulou A, Hingorani AD, Williams DJ. Prospective study of placental angiogenic factors and maternal vascular function before and after preeclamspia and gestational hypertension. Circulation 2010; 122: 478-87.

27. Romero R, Nien JK, Espinoza J, et al. A longitudinal study of angiogenic (placental growth factor) and anti-angiogenic (soluble endoglin and soluble vascular endothelial growth factor receptor-1) factors in normal pregnancy and patients destined to develop preeclampsia and deliver a small for gestational age neonate. J Matern Fetal Neonatal Med 2008; 21: 9-23.

28. Llurba E, Crispi F, Verlohren S. Update on the pathophys iological implications and clinical role of angiogenic fac tors in pregnancy. Fetal Diagn Ther 2015; 37: 81-92.

29. Zeisler H, Llurba E, Chantraine F, et al. Predictive value of the sFlt-1: PIGF ratio in women with suspected preeclampsia. N Engl J Med 2016; 374: 13-22.

30. Verlohren S, Herraiz I, Lapaire O, et al. New gestational phase-specific cutoff values for the use of the soluble fms-like tyrosine kinase-1/placental growth factor ra tio as a diagnostic test for preeclampsia. Hypertension 2014; 63: 346-52.

31. Hoffmann J, Ossada V, Weber M, Stepan H. An intermediate sFlt-1/PIGF ratio indicates an increased risk for adverse pregnancy outcome. Pregnancy Hypertens 2017; 10: $165-70$

32. Rana S, Powe CE, Salahuddin S, et al. Angiogenic factors and the risk of adverse outcomes in women with sus pected preeclampsia. Circulation 2012; 125: 911-9.

33. Stepan H, Hund M, Gencay M, et al. A comparison of the diagnostic utility of the sFlt-1/PIGF ratio versus PIGF alone for the detection of preeclampisa/HELLP syndrome. Hypertens Pregnancy 2016; 35: 295-305.

34. Herraiz I, Llurba E, Verlohren S, Galindo A; Spanish Group for the Study of Angiogenic Markers in Preeclampsia. Update on the diagnosis and prognosis of preeclampsia with the aid of the sFlt-1/ PIGF ratio in singleton pregnancies. Fetal Diagn Ther 2018; 43: 81-9.

35. Verlohren S, Herraiz I, Lapaire Oet al. The sFlt-1/PIGF ratio in different types of hypertensive pregnancy disorders and its prognostic potential in preeclamptic patients. Am J Obstet Gynecol 2012; 206: 58.e1-e8.

36. Klein E, Schlembach D, Ramoni A, et al. Influence of the sFlt-1/PIGf ratio on clinical decision-making in wom en with suspected preeclampsia. Plos One 2016; 11: e0156013.

37. Thadani R, Kisner T, Hagmann $\mathrm{H}$, et al. Pilot study of extracorporeal removal of soluble fms-like tyrosine kinase-1 by dextran sulfate apheresis in preeclampsia. Circulation 2011; 124: 940-50.

38. Thadani R, Hagmann H, Schaarschmidt W, et al. Removal of soluble fms-like tyrosine kinase- 1 by dextran sulfate apheresis in preeclampsia. J Am Soc Nephrol 2016; 27: 903-13. 